\title{
Measured and Estimated Ground Reaction Forces for Multi- Segment Foot Models
}

\author{
Dustin A. Bruening \\ Brigham Young University - Provo, dabruening@byu.edu \\ Kevin M. Cooney \\ Frank L. Buczek
}

Follow this and additional works at: https://scholarsarchive.byu.edu/facpub

Part of the Exercise Science Commons

\section{Original Publication Citation}

Bruening DA, Cooney KM, Buczek FL, Richards JG. Measured and estimated ground reaction forces for multi-segment foot models. J Biomech. 2010 Dec 1;43(16):3222-6. doi: 10.1016/ j.jbiomech.2010.08.003. Epub 2010 Sep 9.

\section{BYU ScholarsArchive Citation}

Bruening, Dustin A.; Cooney, Kevin M.; and Buczek, Frank L., "Measured and Estimated Ground Reaction Forces for Multi-Segment Foot Models" (2010). Faculty Publications. 1625.

https://scholarsarchive.byu.edu/facpub/1625

This Peer-Reviewed Article is brought to you for free and open access by BYU ScholarsArchive. It has been accepted for inclusion in Faculty Publications by an authorized administrator of BYU ScholarsArchive. For more information, please contact ellen_amatangelo@byu.edu. 


\title{
Measured and estimated ground reaction forces for multi-segment foot models
}

\author{
Dustin A. Bruening, Kevin M. Cooney, Frank L. Buczek, James G. Richards
}

\begin{abstract}
Accurate measurement of ground reaction forces under discrete areas of the foot is important in the development of more advanced foot models, which can improve our understanding of foot and ankle function. To overcome current equipment limitations, a few investigators have proposed combining a pressure mat with a single force platform and using a proportionality assumption to estimate subarea shear forces and free moments. In this study, two adjacent force platforms were used to evaluate the accuracy of the proportionality assumption on a three segment foot model during normal gait. Seventeen right feet were tested using a targeted walking approach, isolating two separate joints: transverse tarsal and metatarsophalangeal. Root mean square (RMS) errors in shear forces up to 6\% body weight (BW) were found using the proportionality assumption, with the highest errors (peak absolute errors up to $12 \% \mathrm{BW}$ ) occurring between the forefoot and toes in terminal stance. The hallux exerted a small braking force in opposition to the propulsive force of the forefoot, which was unaccounted for by the proportionality assumption. While the assumption may be suitable for specific applications (e.g. gait analysis models), it is important to understand that some information on foot function can be lost. The results help highlight possible limitations of the assumption. Measured ensemble average subarea shear forces during normal gait are also presented for the first time.
\end{abstract}

Keywords: Ground reaction force; Shear force; Plantar shear; Foot model; Multisegment foot

\section{Introduction}

The traditional single segment foot model used in clinical gait analysis and human movement research is being replaced by more complex multiple segment models (Baker and Robb, 2006); however, the addition of kinetics to these models has been hampered in large part by equipment limitations. In particular, complete ground reaction forces (GRFs comprising normal forces, shear forces, and a free moment) applied to discrete subareas of the foot have been difficult to obtain due to insufficient spatial and temporal resolution (Davis et al., 1998 and Scott and Winter, 1993). 
To overcome current measurement limitations, a few investigators have proposed combining a pressure mat with a force platform and estimating subarea shear forces by assuming that they are distributed in proportion to normal forces (Abuzzahab et al., 1997, Giacomozzi and Macellari, 1997 and MacWilliams et al., 2003). Yavuz et al. (2007) performed the only previously published analysis of this proportionality assumption, testing its ability to predict peak shear stresses under the forefoot in normal and diabetic gait. Using a small, custom-built array of triaxial force sensors, they reported errors as high as $98 \mathrm{kPa}$, suggesting that these were due to local differences in plantar frictional properties and/or intrinsic muscle activities. The proportionality assumption has not been tested across the entire foot (i.e. as applied to a multi-segment foot model), where interactions between foot segments may introduce additional errors. For example, when a vertical load is applied to an arch, shear reaction forces at the bases are directed toward the center of the arch (i.e. in opposite directions) and cancel if measured by a single force platform. Similar situations may exist in the loaded foot at the medial longitudinal arch (Sarrafian, 1987) or between the metatarsals and phalanges. The purpose of the present study was to evaluate the ability of the proportionality assumption to predict shear forces and free moments under a three segment foot model during normal gait. It was hypothesized that opposing shear forces would occur between segments for which the proportionality assumption could not account. Accurate measurement of subarea GRFs will improve multi-segment and finite element foot models, and lead to increased understanding of foot and ankle pathologies.

\section{Methods Data collection}

Seventeen normal, healthy pediatric subjects ( 9 males, 8 females), representing a range of ages (7-18 years, mean 12.6 \pm 3.4 ) and associated foot sizes, volunteered and signed approved consent/assent forms. The right foot of each subject was evaluated. Subjects first walked at a self-selected speed across a floor containing two adjacent AMTI OR6-7-1000 force platforms (Advanced Mechanical Technology Inc., Watertown, MA, USA). Three trials were collected during which the entire foot made contact with a single platform. Next, subjects walked using a three-step targeting approach so that specified portions of the foot contacted the adjacent platforms. This was done for two different joints: (1) transverse tarsal (TT) and (2) metatarsophalangeal (MTP). For the TT trials, the segments were divided approximately at the transverse tarsal joints, so that the rearfoot contacted the first platform while the rest of the foot contacted the second platform. For the MTP trials, the division was just distal to the metatarsal heads, with the hallux and toes isolated on the second platform. For both conditions, a foot outline was drawn on the force platforms for visual guidance, but subjects were instructed to walk as normally as possible and the starting position was adjusted until the appropriate foot placement was achieved. Numerous trials were collected for each condition until at least three were identified with accurate foot placement, which was verified by two video cameras located on either side of the platform division (Fig. 1). 

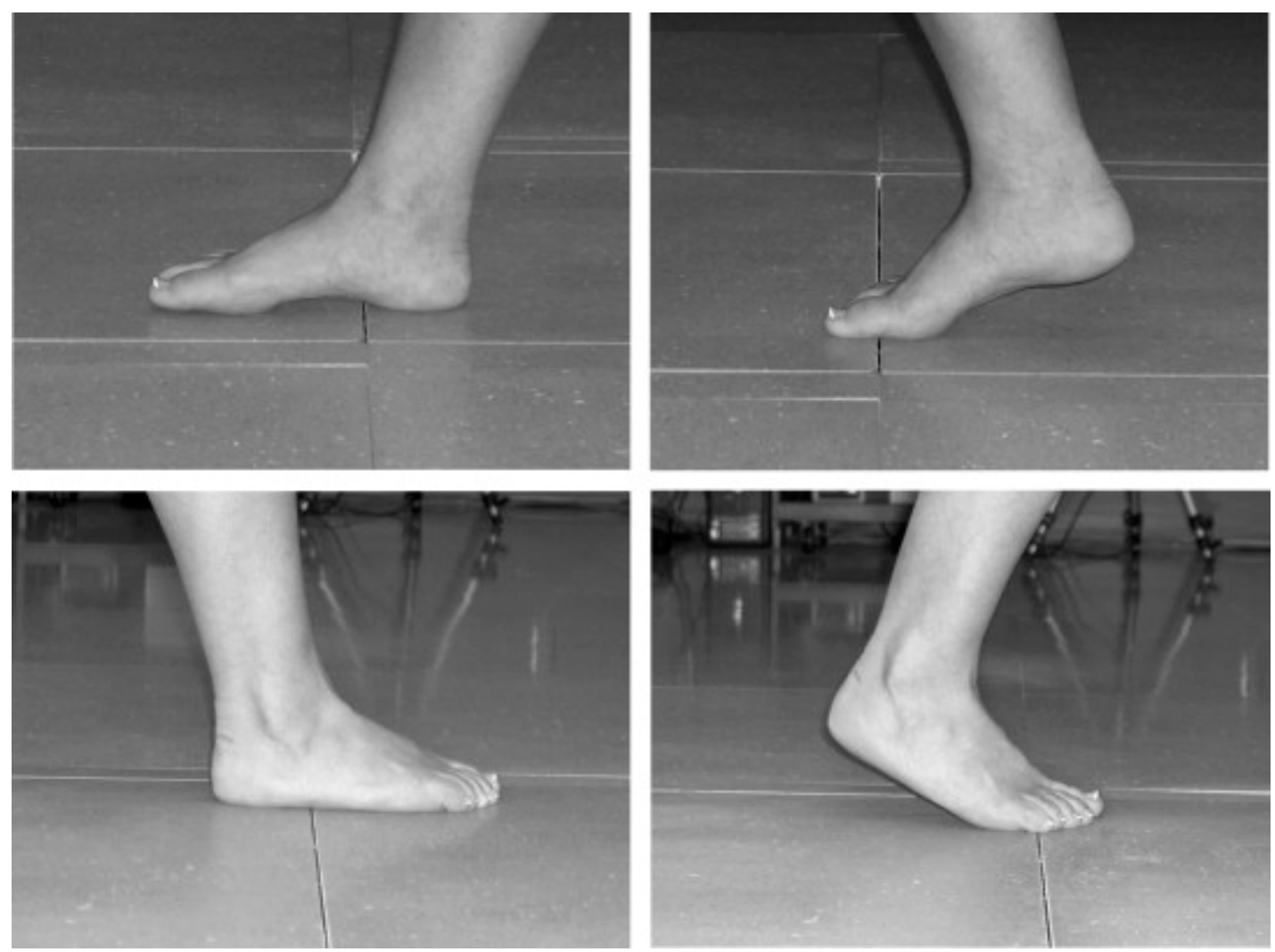

Fig. 1. Example photographs showing foot placement spanning two adjacent force platforms. Left panels: Transverse tarsal (TT) trial with foot divided approximately at the transverse tarsal joints. Right panels: Metatarsal-phalangeal (MTP) trial with foot divided between metatarsals and phalanges.

\section{Data analysis}

Six-channel GRF data were analog low-pass filtered at $1050 \mathrm{~Hz}$ and collected at $1560 \mathrm{~Hz}$. All data processing was performed using a Visual 3D software (C-Motion Inc. Germantown, MD, USA). For the targeted trials, the channels from the two force platforms were combined to create a single virtual platform. Both single and virtual platform GRF data were then filtered using a low pass Butterworth filter (100 Hz cutoff frequency), and a threshold cutoff of $5 \mathrm{~N}$ was applied. The virtual platform was used in conjunction with the normal force components from the individual platforms (i.e. similar to subarea forces created by integrating over a pressure mat) to estimate the shear forces and free moment on each foot segment using the proportionality assumption as per Cowley (2001) and MacWilliams et al. (2003). Curves were time-normalized to stance phase for comparisons between trials. A representative trial for each subject and each joint condition was chosen by first calculating mean GRFs across the three self-selected speed trials. Root mean square (RMS) differences were then calculated between virtual GRF components from the TT and MTP trials and the mean GRF components from the self-selected speed trials. The trial with the smallest RMS differences across all GRF components was chosen 
as representative for that subject and joint. Using the representative trials, RMS errors were computed between the estimated and measured GRFs over time periods in which both segments were in contact with the platform. All forces were normalized by body weight (\% BW) for comparisons between subjects.

\section{Results}

Ensemble averages of measured shear and normal GRF components demonstrated reasonably low variability across subjects (Fig. 2). With few exceptions, errors in estimated shear forces were similar in magnitude and direction across subjects, and mean curves for both measured and estimated forces show typical errors (Fig. 3A-B). RMS errors ranged from 1\% BW to 6\% BW, and were greater in the MTP trials than in the TT trials (Table 1). Peak absolute errors up to 12\% BW were observed in the MTP trials, anteroposterior (A/P) direction, during terminal stance. Mean measured and estimated free moments were more variable than shear and normal forces, and were not representative of all individual moment patterns (Fig. 3C). Free moment RMS errors ranged from 0.001 to $0.027 \mathrm{Nm} / \mathrm{kg}$, with maximum errors up to $0.040 \mathrm{Nm} / \mathrm{kg}$.
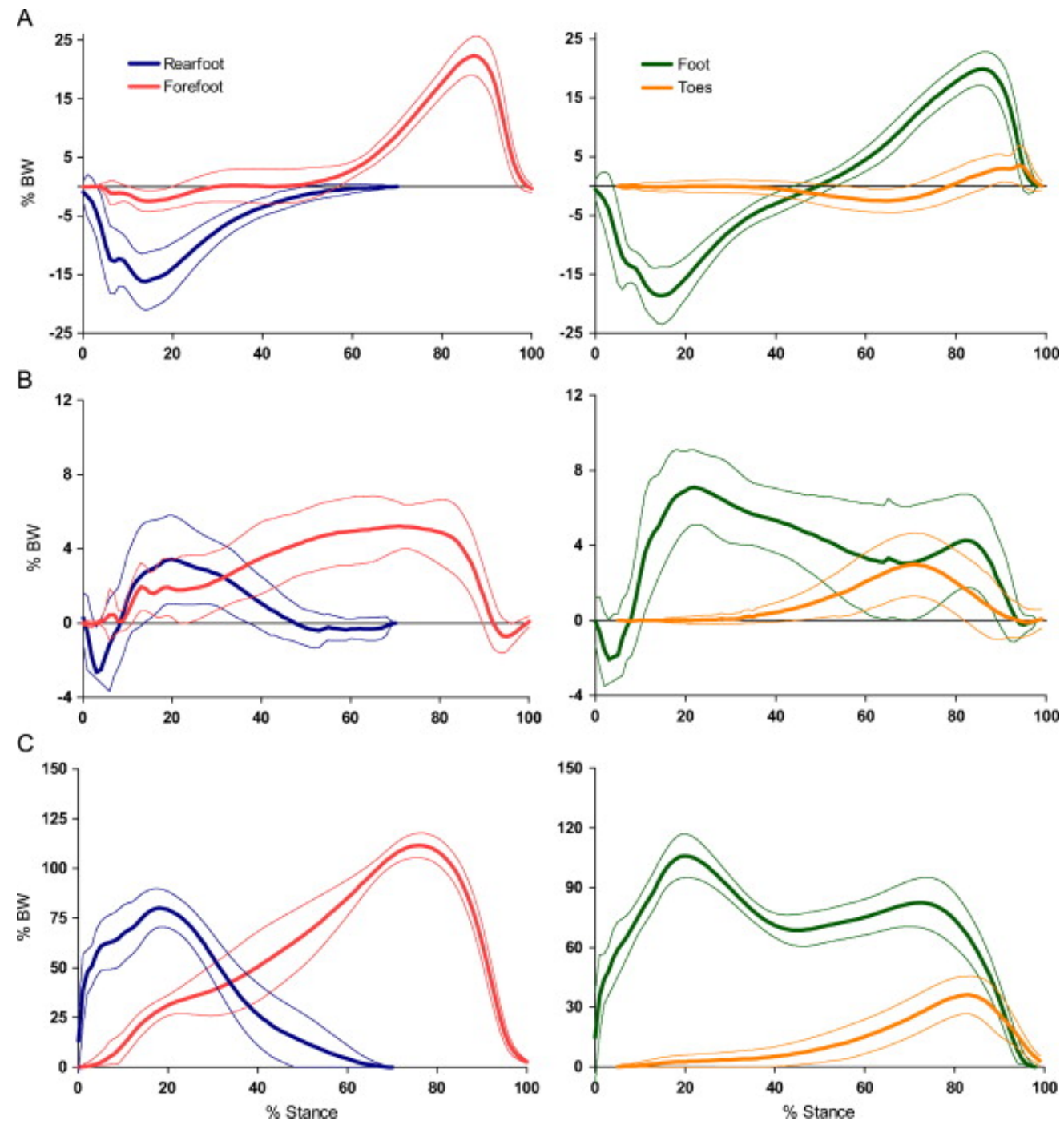
Fig. 2. Ensemble average (mean \pm standard deviation) measured GRFs for each joint condition during normal, targeted gait $(n=17)$. Graphs in the left column are from the TT trials, while graphs in the right column are from the MTP trials.

A
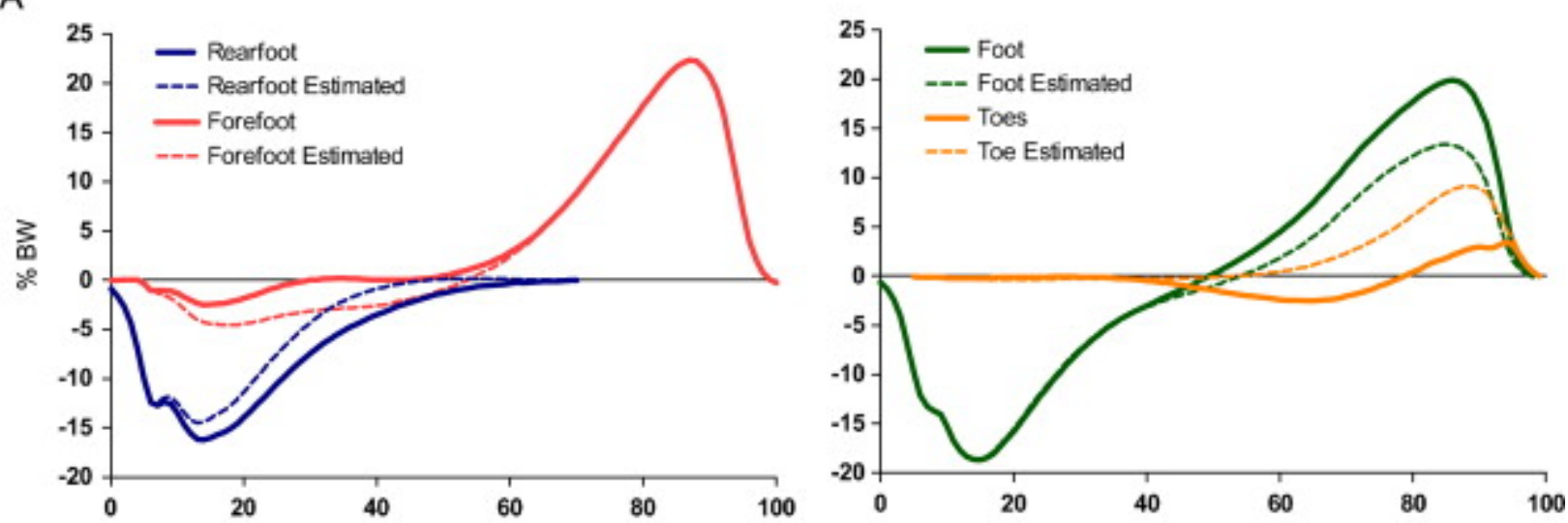

B
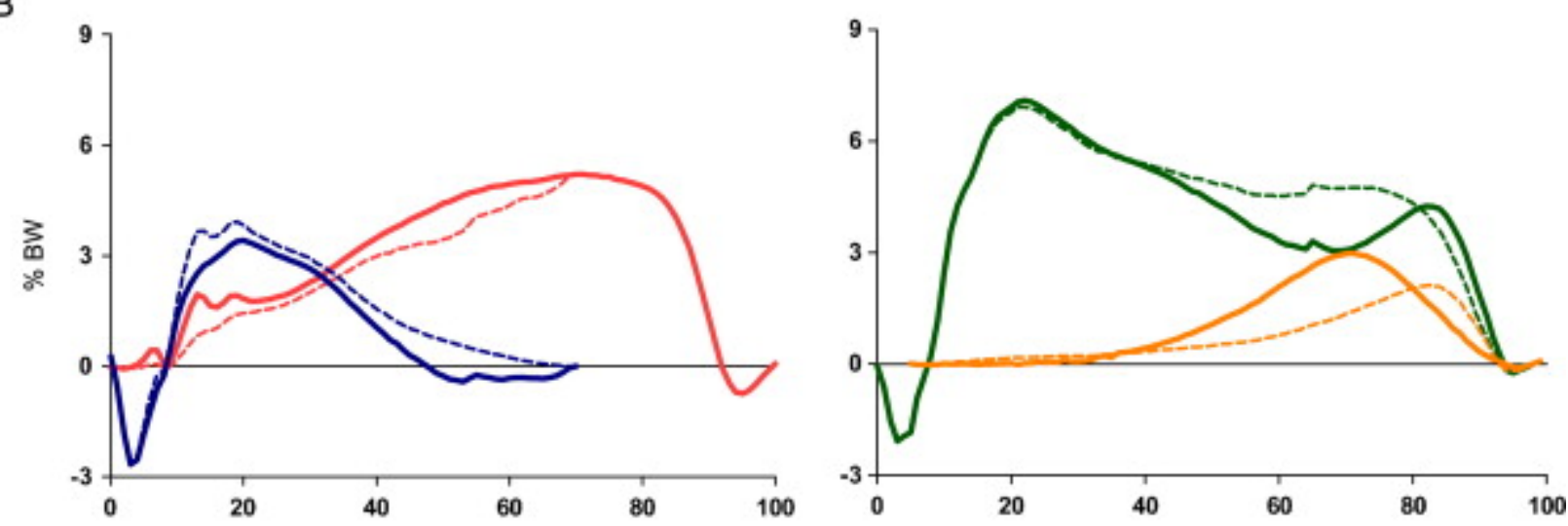

C
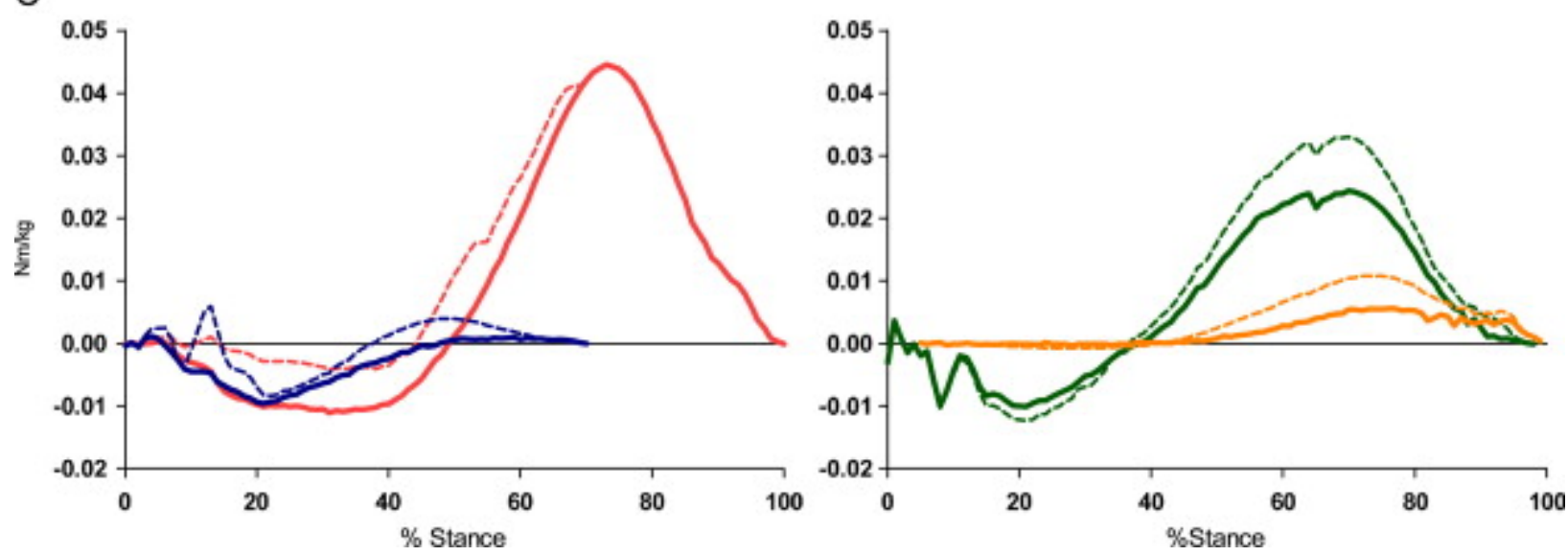

Fig. 3. Mean ( $n=17)$ measured (solided lines) and estimated (dashed lines) shear forces and free moments during normal, targeted gait. Graphs in the left column are from the TT trials while graphs in the right column are from the MTP trials. 
Table 1. Estimated shear force and free moment RMS and maximum errors (mean \pm standard deviation, $n=17$ ). It should be noted that shear force errors on adjacent segments are equal and opposite, while no such relationship exists for the free moments.

\section{Segment}

M/L shear (\% BW) A/P shear (\% BW) Free moment ( $\mathrm{Nm} / \mathrm{kg})$

$\begin{array}{lrrrrrc} & \text { RMS } & \text { Max } & \text { RMS } & \text { Max } & \text { RMSE } & \text { Max } \\ \text { Rearfoot } & 1.3 \pm 0.6 & 2.3 \pm 0.8 & 2.6 \pm 1.1 & 3.6 \pm 1.3 & .009 \pm .004 & .021 \pm .008 \\ \text { Forefoot } & 1.3 \pm 0.6 & 2.3 \pm 0.8 & 2.6 \pm 1.1 & 3.6 \pm 1.3 & .012 \pm .006 & .020 \pm .009 \\ \text { Foot } & 1.2 \pm 0.6 & 2.5 \pm 1.1 & 4.0 \pm 1.6 & 7.8 \pm 3.0 & .007 \pm .003 & .013 \pm .005 \\ \text { Toes } & 1.2 \pm 0.6 & 2.5 \pm 1.1 & 4.0 \pm 1.6 & 7.8 \pm 3.0 & .004 \pm .002 & .009 \pm .005\end{array}$

\section{Discussion}

Combining a pressure mat and a force platform to estimate subarea GRFs has appealed to several investigators because of its simplicity and practicality (Abuzzahab et al., 1997, Giacomozzi et al., 2006, Giacomozzi and Macellari, 1997, Giacomozzi et al., 2000, MacWilliams et al., 2003 and Saraswat et al., 2010) but the underlying proportionality assumption has not been rigorously evaluated. In this study, we used two adjacent force platforms to test the accuracy of the approach as it applies to a three segment foot model.

In the A/P direction, in early to mid-stance, the proportionality assumption slightly underestimated the braking force on the rearfoot, and attributed it instead to the forefoot (Fig. 3A, left panel). Small A/P opposing shear forces were noted in mid-stance, presumably due to collapse of the medial longitudinal arch, but the effect was smaller than hypothesized and did not occur in all subjects (Fig. 2A, left panel). More pronounced A/P opposing forces were seen in late stance, as the toes exerted a small braking force during the initiation of the forefoot roll-over (third rocker). The propulsive contribution of the hallux and toes was consequently overestimated by the proportionality assumption (Fig. 3A, right panel), and peak errors up to $150 \%$ of the measured value were observed in late terminal stance.

In the mediolateral $(\mathrm{M} / \mathrm{L})$ direction, many subjects showed minor, opposing forces during midstance likely due to transverse plane foot rotation (Fig. 3B, left panel). Larger M/L errors were seen between the forefoot and toes throughout terminal stance, but the measured and estimated values were both directed medially (Fig. 3B, right panel), suggesting distinct differences in loading between the segments. Both measured and estimated free moment patterns (Fig. 3C) were more variable than shear forces, and estimation errors varied considerably across subjects and gait cycle. Maximum measured free moments were generally produced by the forefoot, in terminal stance (Fig. 3), and errors up to $250 \%$ of the measured values were observed. 
Giaccomozi and Macellari (1997) suggested accounting for the effects of foot rotation by partitioning the total free moment among the various segments, adjusting the shear forces, and recalculating subarea free moments. This method requires that the sensors be small enough that they can be treated as points (containing only negligible free moments), and therefore cannot be applied or evaluated in this study. It is possible that Giaccomozi's method could reduce some of the errors seen in the $\mathrm{M} / \mathrm{L}$ direction due to foot rotation. However, the largest free moments were generally confined to the forefoot, after the rearfoot was unloaded (Fig. 3C), and Yavuz et al. (2007) actually found greater M/L peak forefoot errors using Giaccomozi’s method compared with the simple version favored by MacWilliams et al. (2003). The additional assumptions required in Giaccomozi's method may result in shear errors that are compounded, rather than reduced. No statements can be made regarding the accuracy of free moments calculated using Giaccomozi’s method.

The split force platform methodology limited force analysis to two of the three segments in a single trial. This allowed the rearfoot and toes to be completely isolated (although separately), but not the forefoot. However, the most important interactions between the forefoot and rearfoot occurred while the force on the toes was small, and similarly, forces on the rearfoot were small or non-existent for the important interactions between the forefoot and toes.

Targeted, or visually guided, walking was used to achieve practical subarea GRF measurements from adjacent force platforms. Previous studies have shown good agreement between targeted and non-targeted ground reaction forces (Grabiner et al., 1995 and Patla et al., 1989) as well as plantar pressures (Bryant et al., 1999, McPoil et al., 1999 and Meyers-Rice et al., 1994). The representative trials were also chosen to minimize differences between the targeted GRFs and the self-selected speed GRFs. Some small differences were noted, with mean vertical RMS differences less than 3\% BW for the shear forces and less than 10\% for the normal forces. However, all GRF patterns were of the same shape and, with very few exceptions, fell within two standard deviations of the group ensemble averages from the self-selected speed trials, suggesting that the presented forces were reasonably representative of normal gait.

Other limitations are also inherent in the use of adjacent force platforms. Foot positioning was verified visually, but not quantified. There may be some increased inter-subject variability in force measurements due to the $\mathrm{A} / \mathrm{P}$ positioning of the foot relative to the force platform division. However, the foot structure is somewhat arch-like at both the TT and MTP joints, and forces under each joint are generally small (Wearing et al., 2001). The MTP joint line runs slightly diagonal to the force platforms, so the fourth and fifth toes contacted both force platforms during some trials. Again, however, the forces on the lesser toes are small in comparison to the force on the hallux (Wearing et al., 2001) and should have minimal effect on the results. There is also a small gap between platforms, but this is less than $3 \mathrm{~mm}$.

Extending the error analysis from shear forces to joint moments using inverse dynamics is straightforward, but due to the need for a full model description and validation, is saved for future work. However, a few comments can be made in this regard. Because the proportionality assumption simply re-distributes the total measured GRF, joint moments proximal to the foot will generally be unaffected by distribution errors. The normal GRF component and center of pressure dominate foot joint moment calculations, and most foot joint moments will likely be 
only minimally affected by small errors in shear force distribution. While the method may therefore be suitable for applications such as clinical gait analysis, more dynamic activities may exacerbate the errors. Analysis of shear forces alone, particularly at the metatarsophalangeal joint(s), reveals that some information on foot function can be lost when using the proportionality assumption. In all applications, it is important to understand the associated limitations, while for more accurate measurements, a device capable of directly measuring subarea GRFs may be needed (Davis et al., 1998 and Mackey and Davis, 2006).

\section{References}

F.S. Abuzzahab, G.F. Harris, S.M. Kidder. A kinetic model of the foot and ankle. Gait and Posture, 5 (2) (1997), p. 148

R. Baker, J. Robb. Foot models for clinical gait analysis. Gait and Posture, 23 (4) (2006), pp. 399-400

A. Bryant, K. Singer, P. Tinley. Comparison of the reliability of plantar pressure measurements using the two-step and midgait methods of data collection. Foot and Ankle International, 20 (10) (1999), pp. 646-650

Cowley, M., 2001. A multi-segment kinematic and kinetic foot model for clinical decision making. M.S. Thesis, University of Utah, Salt Lake City.

B.L. Davis, J.E. Perry, D.C. Neth, K.C. Waters. A device for simultaneous measurement of pressure and shear force distribution on the plantar surface of the foot. Journal of Applied Biomechanics, 14 (1) (1998), pp. 93-104

C. Giacomozzi, M.G. Benedetti, A. Leardini, V. Macellari, S. Giannini. Gait analysis with an integrated system for functional assessment of talocalcaneal coalition. Journal of the American Podiatric Medical Association, 96 (2) (2006), pp. 107-115

C. Giacomozzi, V. Macellari. Piezo-dynamometric platform for a more complete analysis of foot-to-floor interaction. IEEE Transactions on Rehabilitation Engineering, 5 (4) (1997), pp. 322-330

C. Giacomozzi, V. Macellari, A. Leardini, M.G. Benedetti. Integrated pressure-force-kinematics measuring system for the characterisation of plantar foot loading during locomotion. Medical and Biological Engineering and Computing, 38 (2) (2000), pp. 156-163

M.D. Grabiner, J.W. Feuerbach, T.M. Lundin, B.L. Davis. Visual guidance to force plates does not influence ground reaction force variability. Journal of Biomechanics, 28 (9) (1995), pp. 1115-1117

J.R. Mackey, B.L. Davis. Simultaneous shear and pressure sensor array for assessing pressure and shear at foot/ground interface. Journal of Biomechanics, 39 (15) (2006), pp. 2893-2897

B.A. MacWilliams, M. Cowley, D.E. Nicholson. Foot kinematics and kinetics during adolescent gait. Gait and Posture, 17 (3) (2003), pp. 214-224

T.G. McPoil, M.W. Cornwall, L. Dupuis, M. Cornwell. Variability of plantar pressure data. A comparison of the two-step and midgait methods. Journal of the American Podiatric Medical Association, 89 (10) (1999), pp. 495-501

B. Meyers-Rice, L. Sugars, T. McPoil, M.W. Cornwall. Comparison of three methods for obtaining plantar pressures in nonpathologic subjects. Journal of the American Podiatric Medical Association, 84 (10) (1994), pp. 499-504

A.E. Patla, C. Robinson, M. Samways, C.J. Armstrong. Visual control of step length during overground locomotion: task specific modulation of the locomotor synergy. Journal of Experimental Psychology: Human Perception and Performance, 15 (1989), pp. 603-617

Saraswat, P., Andersen, M.S., Macwilliams, B.A., 2010. A musculoskeletal foot model for clinical gait analysis. In: Proceedings of the 2nd Joint Meeting of the European Society of Movement Analysis for Adults and Children and the Gait and Clinical Movement Analysis Society, Miami, FL. 
S.K. Sarrafian. Functional characteristics of the foot and plantar aponeurosis under tibiotalar loading. Foot and Ankle, 8 (1) (1987), pp. 4-18

S.H. Scott, D.A. Winter. Biomechanical model of the human foot: kinematics and kinetics during the stance phase of walking. Journal of Biomechanics, 26 (9) (1993), pp. 1091-1104

S.C. Wearing, S.R. Urry, J.E. Smeathers. Ground reaction forces at discrete sites of the foot derived from pressure plate measurements. Foot and Ankle International, 22 (8) (2001), pp. 653-661

M. Yavuz, G. Botek, B.L. Davis. Plantar shear stress distributions: comparing actual and predicted frictional forces at the foot-ground interface. Journal of Biomechanics, 40 (13) (2007), pp. 30453049

NIOSH disclaimer: the findings and conclusions in this report are those of the authors and do not necessarily represent the views of the National Institute for Occupational Safety and Health. 